\title{
Global optimization of energy and production in process industries: a genetic algorithm application
}

\author{
Amâncio Santos ${ }^{\mathrm{a}, \mathrm{b}, *, 1}$, António Dourado ${ }^{\mathrm{b}}$ \\ a Instituto Superior de Engenharia do, Instituto Politécnico de Coimbra, Quinta da Nora, Apartado 10057, 3030 Coimbra, Portugal \\ ${ }^{\mathrm{b}}$ Centro de Informática e Sistemas da, Universidade de Coimbra, DEI, Pólo II, Pinhal de Marrocos, 3030 Coimbra, Portugal
}

Received 20 April 1998; accepted 11 November 1998

\begin{abstract}
The process industries exhibit an increasing need for efficient management of all the factors that can reduce their operating costs, leading to the necessity for a global multi-objective optimization methodology that will enable the generation of optimum strategies, fulfilling the required restrictions. In this paper, a genetic algorithm is developed and applied for the optimal assignment of all the production sections in a particular mill in the kraft pulp and paper industry, in order to optimize energy the costs and production rate changes. This system is intended to implement all programmed or forced maintenance shutdowns, as well as all the reductions imposed in production rates. (C) 1999 Elsevier Science Ltd. All rights reserved.
\end{abstract}

Keywords: Production control; Scheduling algorithms; Genetic algorithms; Global optimization; Pulp industry

\section{Introduction}

Plants in the continuous production industries can be described as groups of departments, each responsible for some specific operations and separated by intermediate buffers. The production of kraft pulp and paper is one of such industries.

Consider the notation of Fig. 1, suggested in (Dourado and Santos 1993), where buffer $j$, with level $x_{j}$ $(j=1, \ldots, m)$, receives the production from the department $i$, working at rate $u_{i}(i=1, \ldots, n)$ units, and delivers the raw material to department $i+1$, working at rate $u_{i+1}$ units; $b_{j, i+1} \cdot u_{i+1}$ units are consumed from buffer $j$ for each unit of production $u_{i+1}$. This work is based on the case study of the flowsheet of Centro Fabril de Viana da Portucel, represented in Fig. 5.

Pulp mills (and in general the continuous production industry mills) are complex systems, where shutdowns

\footnotetext{
* Correspondence address. Instituto Superior de Engenharia do, Instituto Politécnico de Coimbra, Quinta da Nora, Apartado 10057, 3030 Coimbra, Portugal. Tel.: + 35139790 200; fax: + 35130701 266; e-mail: amancio@isec.pt.

${ }^{1}$ Partially financed by JNICT/PRAXIS XXI program. Industrial data given by Eng. J. Amaral, Portucel, Viana do Castelo, Portugal.
}

and disturbances are propagated throughout the plant and influence the whole mill. This may lead to mass and energy losses due to transient incorrect chemical dosing, and consequently to production losses by breakdowns in the quality levels. The task of scheduling must minimize these effects.

\section{The production scheduling}

The stock equation (1) represents the overall discrete model for the production coordination, where $B$ is the mass balance matrix, and control vector $u$ and state vector $x$ are the departments' production rates and the intermediate-level buffers, respectively. $T$ is the discretization interval, $N$ is the number of discrete planning intervals and $k=0, \ldots, N-1$ :

$x(k+1)=x(k)+B \cdot T \cdot u(k)$.

Both control $u$ and state $x$ are physically constrained by

$$
\begin{aligned}
& 0 \leqslant u_{\min }(k) \leqslant u(k) \leqslant u_{\max }(k) \leqslant U_{\max }, \\
& 0 \leqslant x_{\min }(k) \leqslant x(k) \leqslant x_{\max }(k) \leqslant X_{\max } .
\end{aligned}
$$

In the flowsheet presented here there are three departments that exhibit some different behaviors from the rest, 


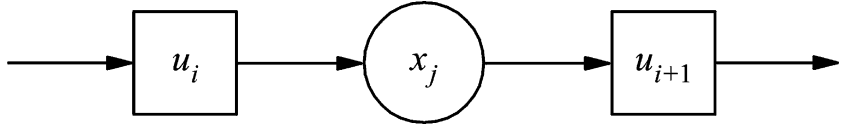

Fig. 1. Flowsheet example with two departments and one buffer.

and therefore require special attention: the water (collection and treatment) department, the auxiliary boiler and the turbogenerator.

The water department produces filtered water for consumption in the various mill departments, and so the production rate is dependent on the rest of the mill. This situation leads to a representation of the water production (4), where $F W$ is the filtered water production and $D_{\text {water }}$ is the water balance matrix:

$F W=D_{\text {water }} \cdot u$.

The task of the auxiliary boiler, together with the recovery boiler, is to produce high-pressure steam (HPS) (the recovery boiler also produces green liquor). The two boilers must fulfil the requirements of HPS in the mill. The mill also needs medium-pressure steam (MPS) and low-pressure steam (LPS) in several sections, namely the paper machine, the pulp mill, the evaporation, the causticizing, and the energy sector. Eqs. (6) and (5) express the relation between the input (HPS) and the output (MPS, $L P S$, condensed water and electrical energy) in the turbogenerator, where HPS, MPS, LPS and CW (condensed water) are given in $\mathrm{kg}$, and the electrical energy $\left(E E_{\text {trbgnr }}\right)$ is given in $\mathrm{kWh}$ :

$$
\begin{aligned}
E E_{\mathrm{trbgnr}}= & (M P S \cdot 77.5+L P S \cdot 117.5 \\
& +C W \cdot 178.0) \frac{0.7}{860.5},
\end{aligned}
$$

$$
H P S=M P S+L P S+C W .
$$

By an analysis of the production values, the turbogenerator production rates are kept at the minimum to maintain the needed output flow of MPS and LPS. Therefore, the flow of condensed water is as low as possible, and a statistical analysis reveals a value of approximately $4.6 \%$ of the HPS consumed in the turbogenerator. The high cost of the fuel consumed in the auxiliary boiler is responsible for this situation (the organic combustible is not enough to produce the steam). Consequently, the auxiliary boiler production can be given by Eq. (7) where $H P S_{\text {total }}$ is the total production of HPS and $H P S_{\text {recb }}$ is the $H P S$ produced in the recovery boiler:

$H P S_{\text {auxb }}=H P S_{\text {total }}-H P S_{\text {recb }}$.

The $H P S_{\text {total }}$ can be given by Eq. (8), where $H P S_{\text {papm }}$ is the high-pressure steam consumption of the paper machine, and $H P S_{\text {trbgnr }}$ is the HPS consumed in the turbogenerator

$H P S_{\mathrm{total}}=H P S_{\mathrm{trbgnr}}+H P S_{\mathrm{papm}}$.
As $H P S_{\text {trbgnr }}$ equals the sum of MPS, LPS and $C W$ produced in the turbogenerator (6), after some calculations $H P S_{\text {total }}$ is given by Eq. (9), where $L P S_{\text {mass }}$ and $M P S_{\text {mass }}$ are, respectively, the low- and medium-pressure steam consumption by the mass chain of the mill, $b_{L P S}^{\mathrm{auxb}}$ and $b_{M P S}^{\mathrm{auxb}}$ are the low- and medium-pressure specific consumption by the auxiliary boiler, and $H P S_{\text {papm }}$ is the high-pressure steam consumption by the paper machine:

$H P S_{\text {total }}=\frac{N u m}{\text { Den }}$,

with

$$
\begin{aligned}
N u m=[ & M P S_{\mathrm{mass}}+L P S_{\mathrm{mass}}-\left(b_{L P S}^{\mathrm{auxb}}+b_{M P S}^{\mathrm{auxb}}\right) H P S_{\mathrm{recb}} \\
& \left.+H P S_{\mathrm{papm}}\right] \frac{1}{0.954}, \\
\text { Den }=1 & -\frac{1}{0.954} \cdot\left(b_{L P S}^{\mathrm{auxb}}+b_{M P S}^{\mathrm{auxb}}\right) .
\end{aligned}
$$

The electrical energy production of the turbogenerator, after the elimination of the condensed water, is given by Eq. (10) where $L P S_{\text {total }}$ and $M P S_{\text {total }}$ are described by Eqs. (11) and (12):

$$
\begin{aligned}
& E E_{\text {trbgnr }}=70.017 \cdot M P S_{\text {total }}-102.566 \cdot L P S_{\text {total }}, \\
& L P S_{\text {total }}=L P S_{\text {mass }}+b_{L P S}^{\text {auxb }} \cdot H P S_{\text {auxb }}, \\
& M P S_{\text {total }}=M P S_{\text {mass }}+b_{M P S}^{\text {auxb }} \cdot H P S_{\text {auxb }} .
\end{aligned}
$$

The electrical energy bought from the public power system is computed by Eq. (13):

$E E_{E D P}=E E_{\text {total }}-E E_{\text {trbgnr }}$.

The total electrical energy consumed in the mill must be minimized by Eq. (14), where $B_{E E}$ is the energy balance matrix:

$E E_{\text {total }}=B_{E E} \cdot T \cdot u$.

\section{Mathematical formulation}

There are some issues that should be addressed in the production scheduling, as stated in Leiviskä (1982) and in Uronen (1981):

(1) the final production must be accomplished within the planning time horizon, since delays in delivery times lead to economic losses;

(2) the storage capacities should be used in order to avoid over- and underflows and also to

(3) avoid production-rate changes, as these are responsible for additional costs due to efficiency breakdowns in almost all departments;

(4) the maintenance shutdowns should be carefully planned so as to benefit the entire mill;

(5) the end of one schedule plan should be seen as the beginning of the next one, and therefore the final storage levels should be pre-determined; 
(6) some attention should be paid to the energy consumption, since the pulp and paper industry is highly energy-demanding.

The mathematical formulation must take account of all the aspects mentioned above. From these, it is essential to distinguish between objectives and constraints.

From the above statements, it is seen that in this problem two criteria are needed, given by Eqs. (15) and (16) where $\operatorname{ch}(k, i)$, as stated in Monteiro (1992), is the production-rate change function (department $i$ and instant $k$ ) defined in Eq. (17):

Obj $1=\min \sum_{k=0}^{N-1}\left\{B_{E E} \cdot T \cdot u(k)\right\}$,
Obj $2=\min \sum_{k=1}^{N-1} \sum_{i=1}^{n} \operatorname{ch}(k, i)$,
$\operatorname{ch}(k, i)=\left\{\begin{array}{l}1 \Leftarrow u_{i}(k) \neq u_{i}(k-1), \\ 0 \Leftarrow u_{i}(k)=u_{i}(k-1) .\end{array}\right.$

The formulation will be completed by a constraint set definition:

- the accomplishment of final production, during the planning time horizon, must corroborate Eq. (18), where $x_{\text {mpap }}$ stands for the paper machine buffer level and $K_{\text {fpap }}$ represents the finished paper needed:

$x_{\text {mpap }}(N-1)-x_{\text {mpap }}(0)=K_{\text {fpap }}$;

- the planned maintenance shutdowns and the production restrictions expressed by Eq. (2);

- the minimum and maximum safety limits of all storage buffers, as stated in Eq. (3);

- the buffers' final state, which should be pre-determined, as in Eq. (19) where $x_{\text {final }}$ represents the intended final state of the buffers;

$x(N)=x_{\text {final }}$

- the contracted electrical power, which is time variant, should not be exceeded, as in Eq. (20) where $P_{c}(k)$ is the contracted power limit:

$E E_{E D P}(k) \leqslant P_{c}(k)$.

\section{The genetic algorithm}

The optimization of objectives (15) and (16) cannot be achieved by traditional methods since it is a mixed integer problem. However, since genetic algorithms are able to solve mathematically ill-defined problems, they are a tool of great potential. In this work a GA multicriteria approach is used, based on constraint-handling techniques.

Several methods exist for handling constraints by genetic algorithms in optimization problems. The technique used here (Michalewicz, (1994) is based on preserving the feasibility of solutions by using specialized operators that are closed on the feasible part of the search space. These operators (crossover and mutation) transform feasible solutions into other feasible solutions. The basic idea behind this method lies (i) in the elimination of the equalities present in the constraint set and, (ii) in the use of specific operators that guarantee that individuals are kept inside the feasible space.

GAs have been used particularly in single-objective problems; nevertheless, most of the practical applications exhibit more than one objective to be attended to. In this work, the Pareto ranking method is used in order to properly select the next generation. This technique, which makes use of the definition of Pareto optimality, was first introduced by Goldberg (1989) and later redefined as a slightly different scheme in Fonseca and Fleming (1993). As proposed by Fonseca, an individual's rank corresponds to the number of individuals in the current population by which it is dominated; therefore, the heavily dominated individuals are given a worse chance of reproduction. This process ends with the fitness assignment by interpolating from the best individual to the worst, usually according to an exponential function, but possibly also using other types. Here the function expressed in Eq. (21) was used, where $P$ is the rank of the best individual, and $0<c<1$ is a constant:

$f_{i}=\frac{c-1}{c^{P}-1} c^{P-i} ; \quad i \in\{1, \ldots, P\}$.

The crossover and mutation operators employed in this algorithm were chosen from those found in the literature, and which, by simulation, proved to be the set with the best convergence time and with the best diversification in the trade-off surface. The uniform crossover is based on Syswerda (1989) and Spears and De Jong (1991), where, at instant $k$, two vectors with dimension $m, x_{k}^{a}$ and $x_{k}^{b}$, exchange genes $i$ with each other; that is, $x_{k}^{a(i)}$ and $x_{k}^{b(i)}$ for $i=1,2, \ldots, m$, with probability $p$. Fig. 2 represents this crossover.

The mutation phase is formed by a set of four strategies: uniform, boundary, non-uniform (Michalewicz, 1994) and exchange mutations. Let $C=\left(c_{1}, \ldots, c_{i}, \ldots, c_{l}\right)$ be a chromosome of length $l$, and let $c_{i} \in\left[a_{i}, b_{i}\right]$ be the gene to which the mutation operator will be applied resulting in gene $c_{i}^{\prime}$; then in the uniform mutation $c_{i}^{\prime}$ is

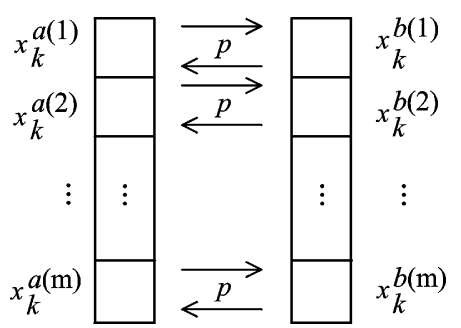

Fig. 2. Uniform crossover with probability $p$. 
a random value, according to a uniform probability distribution, from $\left[a_{i}, b_{i}\right]$. In the boundary mutation $c_{i}^{\prime}$ is either $a_{i}$ or $b_{i}$, with equal probability. In the nonuniform mutation, if $g_{\max }$ is the maximum number of generations, $c_{i}^{\prime}$ is given by (22), where $\alpha \in\{0,1\}$ is a random binary digit, $\Delta(k, y)=y \cdot \beta\left(1-k / g_{\max }\right)^{b}, \beta$ is a random number from the interval $[0,1]$, and $b$ is a parameter determining the dependence degree in the number of generations

$c_{i}^{\prime}=\left\{\begin{array}{l}c_{i}+\Delta\left(k, b_{i}-c_{i}\right) \Leftarrow \alpha=0, \\ c_{i}-\Delta\left(k, c_{i}-a_{i}\right) \Leftarrow \alpha=1 .\end{array}\right.$

Finally, in an exchange mutation, two consecutive genes $c_{i}$ and $c_{i+1}$ are exchanged for each other. This last type can be seen as a particular case of uniform mutation, where interval $\left[a_{i}, b_{i}\right]$ is simply $c_{i+1}$ and $\left[a_{i+1}, b_{i+1}\right]$ is $c_{i}$.

The stochastic universal sampling is used in this work since it is considered the standard algorithm for sampling, which exhibits null distortion and minimum spread. For the reinsertion the elected mechanism was the generational reproduction (Syswerda, 1991) where the whole population is replaced in each generation.

The scheme of sharing was introduced in Goldberg and Richardson (1987), known as fitness sharing, and its main purpose is the distribution of the population in a set of niches in the search space. Use of this procedure eliminates the existence of similar individuals that would lead to redundancy, enemy of diversity. Eq. (23) represents the shared fitness function, where $n n_{i}$ is the niche number of individual $i$, as given in Eq. (24):

$f_{i}^{\text {share }}=\frac{f_{i}}{n n_{i}}$,

$n n_{i}=\sum_{j \in P} \operatorname{Sh}(d(i, j))$.

Function $d(i, j)$ enables the computation of the distance between individuals $i$ and $j$, and represents the distance

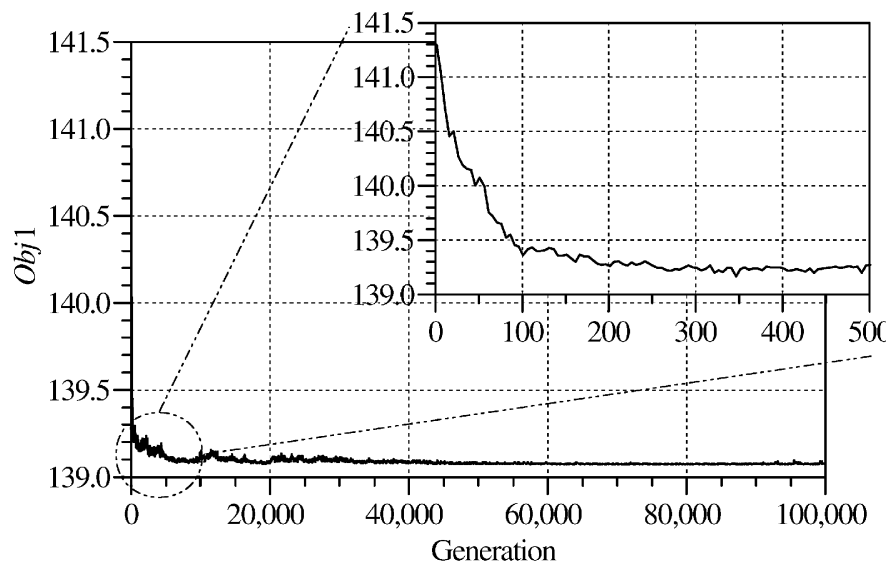

between the vectors formed by all the objective functions in the multicriteria problem. $\mathrm{Sh}(d)$ is the sharing function as expressed by Eq. (25). $\sigma_{\text {share }}$ represents the niche radius which, as stated in Fonseca and Fleming (1993) can be determined by Eq. (26), where $n$ is the number of objectives, $D_{1 i}=M_{i}-m_{i}+\sigma_{\text {share }}, \quad D_{2 i}=M_{i}-m_{i}, \quad m$ and $M$ are the minimum and the maximum of all objectives from the non-dominated set, $Q=\left(d / \sigma_{\text {share }}\right)^{\alpha_{\text {share }}}$ and $\alpha_{\text {share }}$ is a positive real:

$$
\begin{aligned}
& \operatorname{Sh}(d)=\left\{\begin{array}{l}
1-Q \Leftarrow d \leqslant \sigma_{\text {share }}, \\
0 \quad \begin{array}{l} 
\\
0
\end{array} \sigma_{\text {share }},
\end{array}\right. \\
& N \cdot \sigma_{\text {share }}^{n-1}-\frac{\prod_{i=1}^{n} D_{1 i}-\prod_{i=1}^{n} D_{2 i}}{\sigma_{\text {share }}}=0 .
\end{aligned}
$$

Once the sharing scheme has been applied to the population, the crossover between individuals belonging to different niches may result in descendants in any niche. The mating restriction scheme (Deb and Goldberg, 1989) involves the parameter $\sigma_{\text {mate }}$ which is quite similar to $\sigma_{\text {share }}$. The simplest mechanism using this approach is the mating radius which chooses as the second progenitor an individual from the mating pool at a distance less than $\sigma_{\text {mate }}$ from the first progenitor. If none are in this situation, then a random individual is chosen.

\section{Application to the mill, and some simulation results}

With the simplifications introduced in Section 2, three out of the 10 mill departments can be determined subsequently; therefore, the scheduling problem is formed by seven departments. A discretization interval of $4 \mathrm{~h}$ is used, in a planning horizon of $48 \mathrm{~h}$, which leads to 84 system variables. Each chromosome is then coded as real multiparameters, constructed from the concatenated codes. The population is composed of 50 individuals, and the initial ones are randomly generated feasible examples.

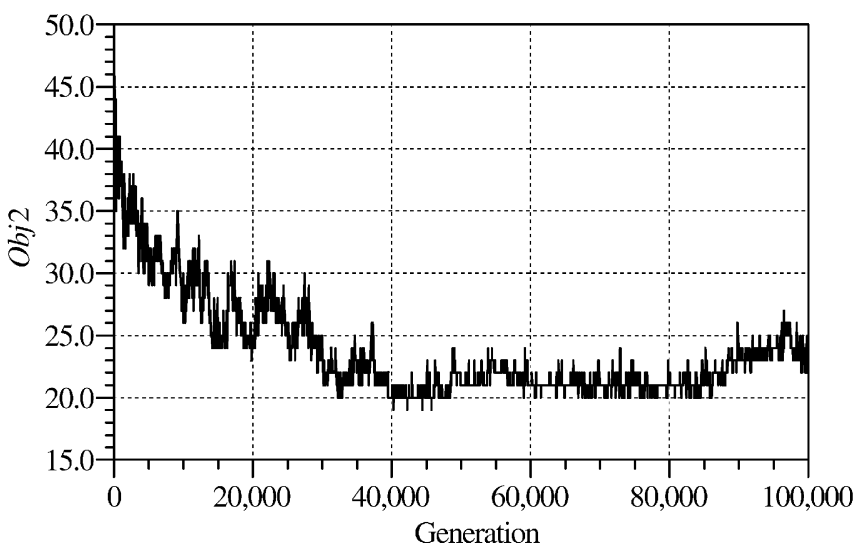

Fig. 3. Evolution of $O b j 1$ and $O b j 2$ across 100,000 generations. 
The initial and final buffer states are constrained to be $50 \%$ of their capacity and the final state for the finished paper is to be $90 \%$ of capacity. A shutdown in the paper mill is also imposed during the third discretization interval, and a reduction to $30 \%$ in the causticizing must occur during the second discretization interval. Due to the limitations of the floating-point representation, a change in a production rate (17) is considered only if it is greater than $2 \%$ of the maximum.

Some simulation results are shown in Figs. 3-5. The evolution of the best individual in the population across 100,000 generations is shown by Fig. 3. Fig. 4 depicts the
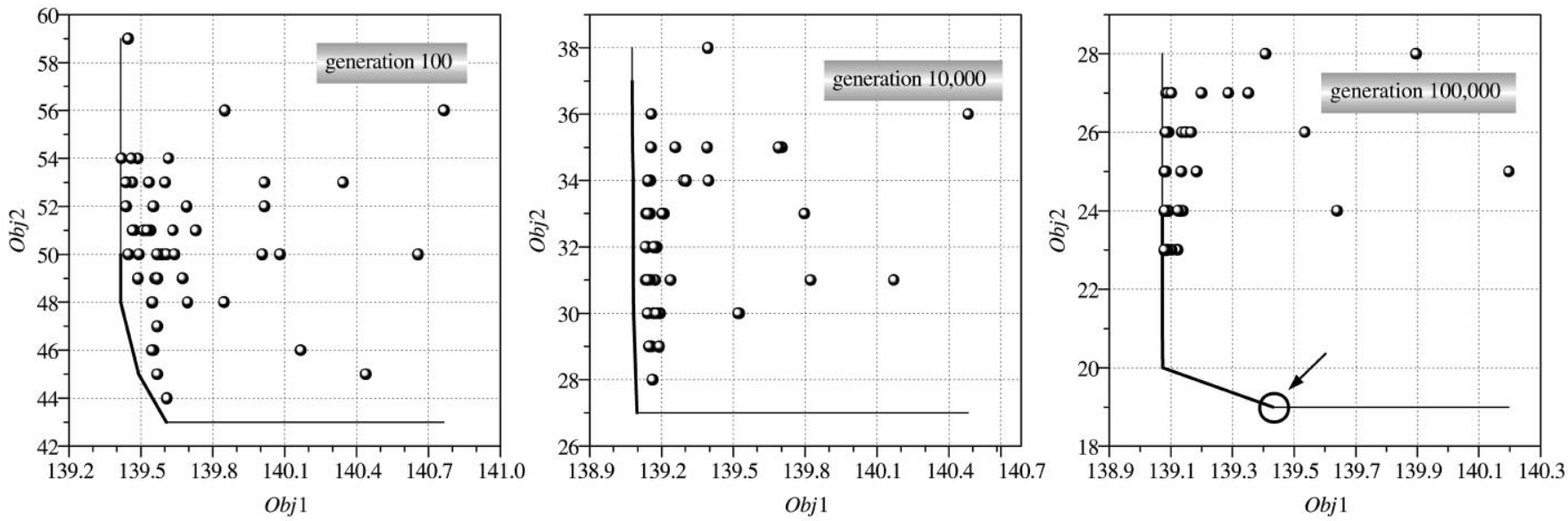

Fig. 4. The population and the cumulative trade-off surface in generations 100, 10,000 and 100,000.

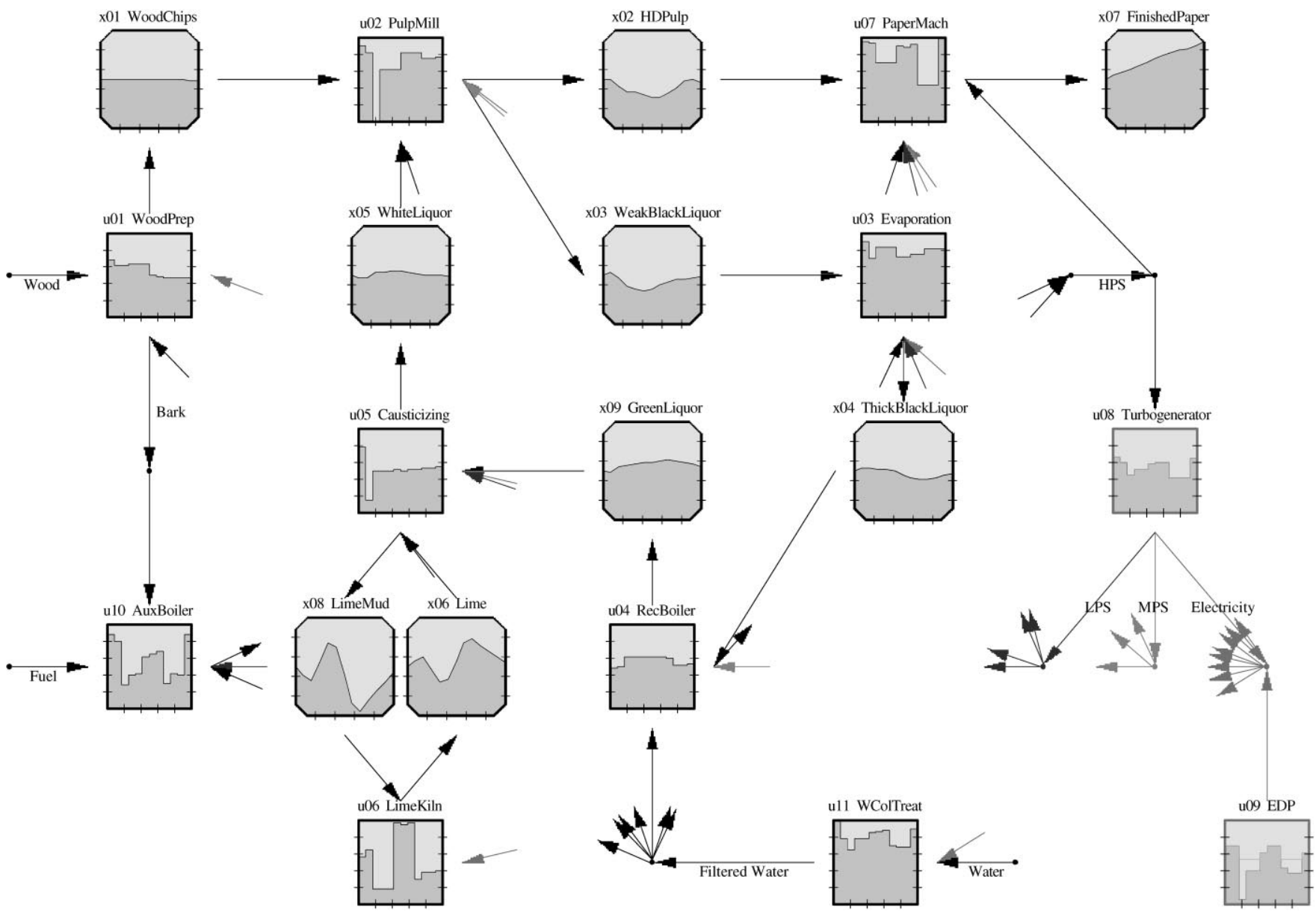

Fig. 5. One solution from the trade-off surface in generation 100,000 . 
population in three different generations as well as the cumulative trade-off surface. Finally, Fig. 5 represents the solution marked in Fig. 4, in generating 100,000, being one of the possible solutions from the optimal Pareto set. These results show optimistic prospects for the potential of the GAs ability to solve this problem.

\section{Conclusions}

The aim of this work is to contribute to the development of an optimal scheduling system for the mass and energy production, with an application to a kraft pulp and paper mill. The dimensions of the problem, its multiobjective characteristic, and the presence of a high-order constraint set preclude the use of (only) traditional optimization techniques. The Pareto ranking method, and a technique that preserves the feasibility of the solutions, were used in a genetic optimization framework. In agreement with other studies (Santos, 1996), these methods and the genetic operators mentioned above (crossover, mutation, sharing and mating restriction) were those that revealed the best convergence time and the best diversification in the trade-off surface.

If a non-linear component were present in the constraint set, the system could be adapted using the proposal in Michalewicz and Nazhiyath (1995). In this way, the technique presented here exhibits a flexibility that is not achieved by traditional optimization methods. Further work will be needed in order to improve the convergence time, which is still the main drawback. Although the literature shows several applications with reasonable computational times in sequential architectures, it could always be possible to go over to parallel technologies, not necessarily using multiprocessors, but using existing resources such as personal computers and data networks.

\section{References}

Deb, K., \& Goldberg, D. (1989). An investigation of niche and species formation in genetic function optimization. In: J.D. Schaffer (Ed.), Proc. 3rd Int. Conf. on Genetic Algorithms (pp. 42-50). George Mason University, Los Altos: Morgan Kaufmann.

Dourado, A., \& Santos, A. (1993). (optimal) rule-based system for production and energy management. Proc. IEEE Int. Conf. on Systems, Man and Cybernetics (Vol. 4, pp. 731-736).

Fonseca, C., \& Fleming, P. (1993). Genetic algorithms for multiobjective optimization: Formulation, discussion and generalization. In: S. Forrest (Ed.), Proc. 5th Int. Conf. on Genetic Algorithms (pp. 416-423). San Mateo, CA:Morgan Kaufmann Publishers.

Goldberg, D. (1989). Genetic Algorithms in Search, Optimization and Machine Learning. Reading. MA: Addison-Wesley.

Goldberg, D., \& Richardson, J. (1987). Genetic algorithms with sharing for multimodal function optimization. In: J. J. Grefenstette (Ed.), Proc. 2nd Int. Conf. on Genetic Algorithms and their Applications (pp. 41-49). Cambridge, MA: Lawrence Erlbaum Associates.

Leiviskä, K. (1982). Short-term production scheduling of the pulp mill. Acta Polytech. Scand. Math. Comput. Sci. 36, 1-80.

Michalewicz, Z. (1994). Genetic Algorithms + Data Structures = Evolution Programs (2nd ed.). Berlin: Springer.

Michalewicz, Z., \& Nazhiyath, G. (1995). Genocop III: A co-evolutionary algorithm for numerical optimization problems with nonlinear constraints. In: D. B. Forgel (Ed.), Proc. 2nd IEEE Int. Conf. on Evolutionary Computation (pp. 647-651). New York: IEEE Press.

Monteiro, P. (1992). Short-term production scheduling: A new and more efficient algorithm and its application to the pulp and paper industry. Inform Decision Technol., 18, 241-263.

Santos, A. (1996). Production and energy optimization in an industrial complex: A genetic algorithm approach (in Portuguese). Master's Thesis. Dep. de Engenharia Informática da Universidade de Coimbra.

Spears, W., \& De Jong, K. (1991). On the virtues of parameterized uniform crossover. In: R. Belew, \& L. Booker (Eds.), Proc. 4th Int. Conf. on Genetic Algorithms. (pp. 230-236). San Mateo, CA: Morgan Kaufman.

Syswerda, G. (1989). Uniform crossover in genetic algorithms. In: J.D. Schaffer, (Ed.), Proc. 3rd Int. Conf. on Genetic Algorithms (pp. 2-9). George Mason University, Los Altos, CA: Morgan Kaufmann.

Syswerda, G. (1991). A study of reproduction in generational and steady state genetic algorithms. In: G. J. E. Rawlins (Ed.), Proc 1st Workshop on Foundations of Genetic Algorithms. (pp. 94-101). San Mateo: Morgan Kaufmann.

Uronen, P. (1981). Production planning systems for integrated paper mills: Tasks and methodology. Pulp Paper Canada, 82(3), 90-94. 